\title{
Ultrasound-accelerated thrombolysis has higher success and limb salvage rates and similar costs compared to catheter-directed thrombolysis for acute limb ischemia
}

\begin{abstract}
Objectives: Ultrasound-accelerated thrombolysis (UAT) has become an alternative to catheter-directed thrombolysis (CDT) for the treatment of acute limb ischemia. The purpose of this study was to compare outcomes and hospital costs for these two treatments.

Methods: The records of all patients treated with UAT (using the EkoSonic Endovascular System, EKOS Corp, Bothell, Washington) and CDT (standard side-hole catheter) at a single institution from January 2007 through December 2010 were reviewed. Patient demographics, treatment times, procedural outcomes, complications, lengths of stay, and hospital economic data were analyzed.

Results: A total of 85 patients were treated; 43 underwent UAT and 42 had CDT. Both treatment groups had similar comorbidities and prior vascular procedures. Overall, $85.9 \%$ had a previous vascular procedure with 52 prosthetic bypass grafts. UAT was successful in $41 / 43$ patients $(95.3 \%)$, whereas CDT was successful in $28 / 42(66.7 \%, \mathrm{P}=.0019)$. Median total treatment time for the UAT group was 21.29 hours versus 56.53 hours for the CDT group $(\mathrm{P}=.000)$. Length of stay was also less for the UAT group (5.65 vs 8.26 days, $\mathrm{P}=.027)$. $30-\mathrm{d}$ amputation rate was significantly higher in the CDT group $(42.9 \%$ vs $18.6 \%, \mathrm{P}<.01)$. There was a significantly higher bleeding rate in the CDT $(23.8 \%$ vs $4.7 \%, \mathrm{P}<.01)$. Total procedural costs were similar for both groups $(\$ 18,270$ for UAT vs $\$ 16,650$ for CDT, $\mathrm{P}=.366)$, as were total admission direct costs $(\$ 19,556$ for UAT vs $\$ 18,520$ for CDT, $\mathrm{P}=.685)$.

Conclusion: UAT has higher success rates, shorter treatment times, higher limb salvage, less bleeding complications, and shorter lengths of stay than patients undergoing CDT. Despite the favorable clinical outcome for UAT, total procedural costs and total admission direct costs remain similar. Our institution has chosen to employ standardized techniques using UAT in order to improve outcomes and decrease complications in the future.
\end{abstract}

Volume 7 Issue 2 - 2016

\author{
Litzendorf M,' Satiani B, ${ }^{2}$ Bline $\mathrm{K},{ }^{3}$ Jean Starr ${ }^{2}$ \\ 'Vascular Surgery, University of Maryland, Charles Regional \\ Medical Center, USA \\ ${ }^{2}$ Division of Vascular Diseases \& Surgery, the Ohio State \\ University Wexner Medical Center, USA \\ ${ }^{3}$ Division of Pediatrics, Nationwide Children's Hospital, USA
}

Correspondence: Jean Starr, Division of Vascular Diseases \& Surgery, the Ohio State University Wexner Medical Center, 376 W. IOth Ave, 70I Prior Hall, Columbus, OH, USA, Tel 6I4-2938536, Fax 6|4-293-8902, Email jean.starr@osumc.edu

Received: October 27, 2016 | Published: November 21, 2016

\section{Introduction}

Acute limb ischemia (ALI) has a significant mortality rate and requires urgent evaluation and treatment. ${ }^{1}$ Despite the seemingly high mortality, limb salvage is possible in up to $80 \%$ of patients. ${ }^{2}$ Thrombotic disease now accounts for the majority of ALI, surpassing rates of embolic etiologies. This may be due in part to both the aging western populations susceptible to arterial thrombosis due to underlying atherosclerotic disease, as well as more aggressive management of rheumatic heart disease and treatment with anticoagulation for atrial fibrillation, thereby decreasing the incidence of cardio-embolic phenomenon. However, patients presenting with embolic disease have a higher mortality, while amputation rates have been documented to be higher in patients presenting with in situ thrombosis. ${ }^{3}$

Treatment of ALI has similarly experienced a shift as evolving endovascular technologies are becoming more prevalent. The role of endovascular treatment for ALI has been delineated in several randomized trials. The results of the TOPAS study illustrate equivalent mortality and limb survival for thrombolysis and surgery as initial management for ALI. ${ }^{4}$ Catheter-directed thrombolysis, in contradistinction to systemic thrombolysis, targets the clot burden directly, and often reveals an underlying culprit lesion which may also be treated with endovascular techniques. The use of ultrasound to accelerate thrombolysis has been demonstrated to be safe and effective in multiple in vitro models ${ }^{5,6}$ and is commonly used to treat venous thromboembolic events. ${ }^{7}$

In an era of cost awareness and reimbursement shifts, it is unclear if ultrasound-accelerated thrombolysis (UAT) will be more cost effective and clinically successful than catheter-directed thrombolysis (CDT). The purpose of this study is to compare both outcomes and costs of CDT to UAT.

\section{Methods}

This was a retrospective study conducted at a single academic institution of all consecutive patients undergoing thrombolysis for an acute arterial occlusion from January 1, 2007 through December 31, 2010. After obtaining institutional IRB approval, subjects were identified from a hospital database and office records using the Current Procedural Terminology code corresponding to percutaneous thrombolysis at that time, 37201. Thrombolysis was performed by vascular surgeons using the EkoSonic Endovascular System (EKOS Corp, Bothell, Washington), a lytic catheter system capable of delivering a thrombolytic medication with an ultrasonic core catheter. ${ }^{8}$ The purpose of the ultrasonic core is to loosen the fibrin strands within the thrombus, exposing more plasminogen receptor sites for the lytic agent. Interventional radiologists used a standard multi-side 
hole catheter for delivery of intrathrombus lytic medication. Patients were not excluded from the study based on their length of ischemic symptoms or etiology of the thromboembolic event. Patients selected for thrombolysis either underwent CDT by a vascular interventional radiologist in an interventional suite or UAT performed by a vascular surgeon in the endovascular hybrid operating room. These choices were based on institutional and individual practice patterns at that time. All patients received reteplase, a recombinant form of human tissue plasminogen activator, but there was variability both in the bolus administered and in the infusion rates, due to clinician preference and prior to the establishment of an institutional standardized protocol. Analysis of the total amount of drug used excluded the bolus amount since this data was not reliably available.

Both groups of patients were managed post-procedurally by the vascular surgery team. All patients who received stents were maintained on clopidogrel. Patients who had been previously taking anticoagulants were continued on their medication(s) at discharge. Procedure results for each group were classified as technically successful or unsuccessful based on the re-establishment of inline flow in the occluded segment. Bleeding complications were confirmed with either documentation of retroperitoneal bleed on CT scan, intracranial bleed on CT scan, physical exam disclosing a groin hematoma, or other access site bleeding requiring blood transfusion or other intervention. Treatment times were analyzed using the total infusion time of the thrombolytic drug. "Late" intervention refers to a procedure performed after 30days but within 6months of the index procedure. Patients were followed for 6 months after lytic therapy.

The medical center's clinical and financial databases were used to retrieve patient specific information, including demographics, comorbidities, procedural outcomes, lengths of stay, complications, and costs. Cost data was generated by applying the hospital's ratio of cost to charges (RCC) for DRG charges. The RCC is an institutionally generated figure used to estimate reimbursement by third party payers. The cost of charged items was applied to calculate a DRG specific RCC by knowing the DRG charges. All charges and costs were converted to 2010 U.S. dollars using the Consumer Price Index. Patient demographics, comorbidities and lengths of stay were compared using an ANOVA analysis and Fishers Exact Test. The Mann-Whitney U Test was used to compare outcomes. Complication rates were analyzed using the chi-square test. Statistical significance was set at $\mathrm{P}<.05$.

\section{Results}

\section{Clinical outcomes}

A total of 85 patients received endovascular treatment for ALI between January 1, 2007 and December 31, 2010. 43 patients underwent UAT and 42 received CDT. There was a high prevalence of coronary artery disease (38/85) and history of tobacco use (54/85). Demographics and co-morbidities were similar between the groups with the exception of ongoing tobacco use which was significantly more common in the UAT group (Table 1). Prior vascular surgical procedures for both groups were also similar and listed in Table 2. Overall, 73 patients had prior vascular intervention $(85.9 \%)$ with 69 open vascular surgeries (52 prosthetic bypass grafts) and 4 endovascular interventions. Only $12(14.1 \%)$ had either in situ thrombosis or embolus as the etiology of the occlusion. The duration of ischemic symptoms was recorded in $67 / 85$ patients and was less than 30days in all except one patient. In general, patients were not selected to undergo lytic therapy if their symptom duration was greater than one month.
Table I Demographics and Comorbidities

\begin{tabular}{llll}
\hline & $\begin{array}{l}\text { Number } \\
\text { of UAT } \\
\text { Patients }\end{array}$ & $\begin{array}{l}\text { Number } \\
\text { of CDT } \\
\text { Patients }\end{array}$ & P-Value \\
\hline Total patient \# & 43 & 42 & \\
Mean Age (years) & 59.3 & 59.1 & NS \\
Male gender & 17 & 15 & NS \\
Diabetes & 14 & 16 & NS \\
Hx of Stroke & 1 & 1 & NS \\
Current Smoker & 27 & 5 & P<.00I \\
Former Smoker & 9 & 13 & NS \\
Renal Disease & 9 & 12 & NS \\
COPD & 9 & 7 & NS \\
Coronary Artery Disease & 20 & 18 & NS \\
Hx of Myocardial Infarction & 13 & 11 & NS \\
Hypercoaguability & 6 & 7 & NS \\
\hline
\end{tabular}

Table 2 Type of Prior Intervention

\begin{tabular}{lll}
\hline Type of Surgery & $\begin{array}{l}\text { Number } \\
\text { of UAT } \\
\text { Patients }\end{array}$ & $\begin{array}{l}\text { Number } \\
\text { of CDT } \\
\text { Patients }\end{array}$ \\
\hline No prior surgery & 7 & 5 \\
Vein bypass & 7 & 8 \\
Prosthetic bypass & 27 & 25 \\
Endarterectomy & 0 & 2 \\
Prior endo intervention & 2 & 2 \\
\hline
\end{tabular}

All P-values NS

In the UAT group there was a $95.3 \%$ success rate, versus a $66.7 \%$ success rate in the CDT group $(\mathrm{P}=.0019)$ (Figure 1). $25 / 43$ patients in the UAT group underwent additional endovascular intervention with either balloon angioplasty alone or in combination with stent placement. In the CDT group, only six patients underwent additional endovascular intervention. Of the UAT patients who were successfully lysed, six underwent revision of a bypass or other open revascularization during the same admission, three patients underwent revision within 6 months, while one patient required a late percutaneous intervention to maintain patency. In the CDT group, three patients underwent in-hospital operative intervention following successful lysis, while one patient went on to have surgical intervention within 6months.

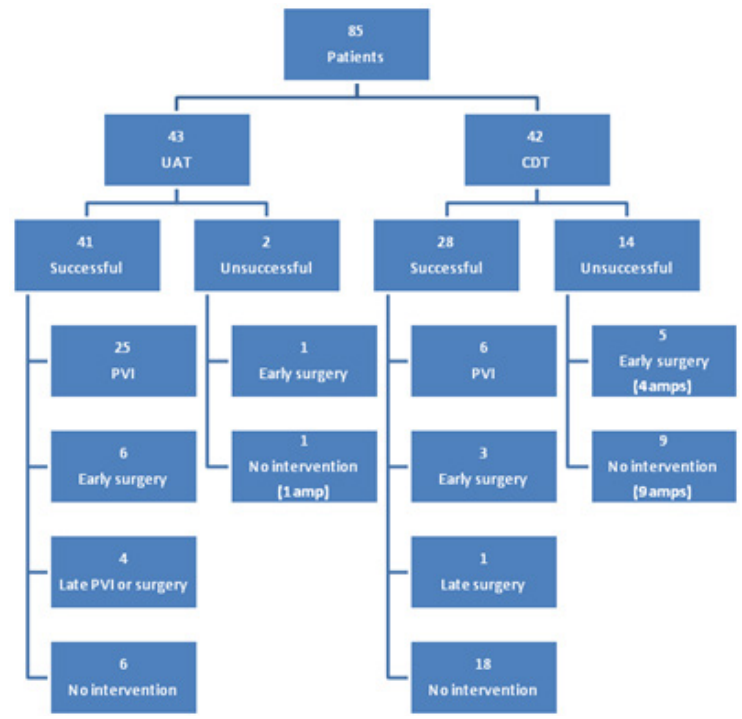

PVI: Peripheral Vascular Intervention;Amp:Above or Below Knee Amputation; "Early": < 30 Days;"Late": < 6Months but more than 30days. 
There were two UAT patients who were not successfully lysed (Figure 1). One patient underwent a tibial embolectomy during the same admission, and was discharged to home with an intact limb. The other unsuccessful UAT patient was not a bypass candidate, was discharged to home, and underwent an amputation within 6months. Of the CDT patients who were not successfully lysed, $92.8 \%$ required amputation within 1month, despite five of fourteen of these patients undergoing an attempt at surgical revascularization.

Eight of ten amputations, distinguished as below or above knee major limb amputation, among the UAT group occurred within 30 days of lysis, while there were 2 late amputations (Table 3 ). The overall amputation rate for the entire population was $32.9 \%$. The rate in the successfully lysed group, irrespective of treatment type, was $20.2 \%(14 / 69)$, compared to $87.5 \%(14 / 16)$ amputation rate in the unsuccessfully lysed group (Figure 1). The 30day amputation rate for the UAT group was $18.6 \%$, versus $42.9 \%$ for the CDT group $(\mathrm{P}=.04)$. All of the CDT group amputations occurred during the initial hospitalization.

Bleeding complications were also found to be significantly higher in the CDT group. There was one intracranial bleed; this occurred in a patient undergoing UAT. This patient subsequently underwent amputation after a prolonged hospital course. The only other UAT bleeding complication was an upper gastrointestinal bleed requiring endoscopy and infusion of a proton pump inhibitor. In contrast, six of the ten bleeding complications in the CDT group were due to access site bleeding events. Two others were from an associated fasciotomy site and two were retroperitoneal bleeds. There was one death in each group, both of which were attributed to multi-system organ failure after prolonged hospitalizations.

All patients undergoing UAT received a 2 unit bolus and were placed on an infusion of 0.5 units per hour. The CDT bolus amount could not be reliably determined from hospital records and infusion rates varied, but in general were close to 0.25 units per hour. The mean infusion drug amount (bolus excluded) used in the UAT group was significantly less than the CDT group (10.64 v 14.58 units, $\mathrm{P}=.034)$. The UAT group had a significantly shorter median treatment time of 21.29hours, versus 56.53hours in the CDT group $(\mathrm{P}=.000)$. Length of stay was also significantly less for the UAT group, whose median stay was 5.65days versus 8.26days in the CDT group $(\mathrm{P}=.027)$

Table 3 Complications

\begin{tabular}{llll}
\hline & $\begin{array}{l}\text { Number } \\
\text { of UAT } \\
\text { Patients } \\
\text { (\# and \%) }\end{array}$ & $\begin{array}{l}\text { Number } \\
\text { of CDT } \\
\text { Patients } \\
\text { (\# and \%) }\end{array}$ & P-Value \\
\hline 30 day amputations & $8(18.6 \%)$ & $18(42.9 \%)$ & $\mathrm{P}<.0 \mathrm{I}$ \\
Late amputations & $2(4.7 \%)$ & 0 & $\mathrm{NS}$ \\
Bleeding & $2(4.7 \%)$ & $10(23.8 \%)$ & $\mathrm{P}<.0 \mathrm{I}$ \\
Death & $\mathrm{I}(0.23 \%)$ & $\mathrm{I}(0.24 \%)$ & $\mathrm{NS}$ \\
\hline
\end{tabular}

\section{Cost analysis}

Cost data is displayed in Table 4. Mean direct costs per patient for the admission were similar between the groups. Limiting the analysis strictly to days while receiving thrombolytic therapy, mean direct costs for UAT are significantly higher than for the CDT group $(\$ 14,167$ v $\$ 11,065 ; \mathrm{P}=.013)$. Further analysis of the cost distribution revealed a higher mean direct cost for operating room use associated with thrombolysis $(\$ 6,674)$ versus interventional radiology suite use $(\$ 4,366)$ for the same procedure, despite shorter treatment times in the UAT group. Numbers of procedures per patient in the operating room or interventional radiology suite were not recorded; therefore "cost per trip" data is not available.

Table 4 Cost Data (\$)

\begin{tabular}{llll}
\hline & $\begin{array}{l}\text { UAT } \\
\text { Group }\end{array}$ & $\begin{array}{l}\text { CDT } \\
\text { Group }\end{array}$ & P-Value \\
\hline Total admission direct costs & 19,556 & 18,520 & 0.685 \\
Direct costs during lysis only & 14,167 & 11,065 & 0.013 \\
Total procedural costs & 18,270 & 16,650 & 0.366 \\
\hline
\end{tabular}

All values in US dollars.

An analysis of pharmacy costs was undertaken to determine the impact of decreased infusion drug used with the EkoSonic Endovascular System. Total pharmacy costs accounted for approximately $1 / 3$ of the total hospital costs for each group. However, when reteplase costs per patient were compared, UAT patients averaged higher mean reteplase costs $(\$ 4,191$ for UAT, $\$ 2,682$ for CDT). Despite these differences, mean total procedural costs between the two groups were similar $(\$ 18,270$ for UAT $\mathrm{v} \$ 16,650$ for $\mathrm{CDT}, \mathrm{P}=.366)$.

\section{Discussion}

ALI is a significant cause of amputation and morbidity. Embolic occlusions may occur in native vessels and thrombotic occlusions in both native vessels and surgically created bypass grafts. Peripheral arterial occlusion, initially thought to be a rare, isolated disease, occurring mostly in an older population, has been shown to occur in a much wider age range with little difference in frequency between men and women, and at a higher rate than previously thought. ${ }^{9}$ Our patient population clearly demonstrates this trend with most patients presenting with prosthetic graft thrombosis. Thus, novel therapeutic modalities are needed to broaden treatment options and overcome current limitations, with a goal to salvage more limbs. Traditional revascularization treatment typically involves open surgical thrombectomy, embolectomy, and bypass operation. Endovascular techniques have rapidly evolved and become not only a viable alternative to open surgery, but often times the preferred modality. However, as new endovascular techniques develop, it has become less clear which endovascular technique is more successful and the most cost effective. Ultrasound-directed thrombolysis is showing promise as a new method of more rapidly re-establishing arterial flow but is not yet considered the treatment of choice.$^{10}$ Our study compared catheterdirected thrombolysis to ultrasound-accelerated thrombolysis in terms of clinical outcomes and costs.

Thrombolysis as a primary modality gained popularity in the 1990's as several randomized studies validated this method for treatment of acute arterial occlusion. The Surgery Versus Thrombolysis for Ischemia of the Lower Extremity (STILE) trial randomized patients with lower extremity arterial occlusions to either immediate surgery or thrombolysis with urokinase or recombinant tissue plasminogen activator. ${ }^{11}$ Patients randomized to surgery were found to have lower rates of ongoing or recurrent ischemia. However, 30day mortality and limb salvage rates were similar. Subgroup analysis demonstrated that thrombolysis was more effective in treating graft occlusions, while patients with native arterial occlusions had lower amputation rates with surgery. In addition, patients randomized to thrombolysis within 14days of their symptoms overall had lower death and amputation rates. The TOPAS trial randomized patients to surgery or to therapy with a recombinant form of urokinase. ${ }^{4}$ Amputation free survival was identical between the two groups at 1year. Although there was no improvement in either survival or limb salvage, TOPAS helped validate thrombolysis for the treatment of acute lower extremity arterial occlusion. 
The traditional thrombolytic method uses a multi-side hole catheter to infuse a thrombolytic medication, such as streptokinase, urokinase, or recombinant tissue plasminogen activator. The alternative method infuses thrombolytics through a special ultrasound driven catheter using high frequency, low power ultrasound. The ultrasound waves increase the permeability of the clot, allowing the drug to have contact with a greater surface area. The mechanism is thought to be acoustic dispersion and microstreaming, generating streams of high flow that enhance lytic drug infusion and increase the kinetics of the lytic agent binding to fibrin, accelerating fibrinolysis. ${ }^{12}$ Proponents argue this decreases the total length of time of drug delivery, which minimizes hemorrhagic side effects of lytic agents that can have devastating outcomes. Patients in our series who received lysis via the EKOS system experienced significantly less bleeding complications than those in the CDT group. This corresponded to significantly less treatment time in the UAT group as well. Additionally, there was less infusion drug utilized in the UAT group. Since bolus drugs amounts were not reliably recorded in the CDT group, it is difficult to relate the increased bleeding complication rate in the CDT group to increased total drug usage. However, there seems to be an association between higher bleeding complication rate and longer treatment time in the CDT group. $60 \%$ of bleeding complications in the CDT group were access site related, potentially related to the longer period an access sheath was left in situ. The recently initiated multi-center DUET study is the first of its kind that will randomize patients with acute limb ischemia to UAT or CDT to assess for differences in therapy time and complication rate. ${ }^{13}$

Several recent reviews of ultrasound accelerated thrombolysis have concluded that it is a promising modality for the management of stroke and pulmonary embolism. ${ }^{14,15}$ The treatment of acute peripheral arterial occlusion with ultrasound accelerated thrombolysis has been evaluated in several recent studies as well. The PARES trial reported a $100 \%$ technical success rate with no amputations or death in 25 patients and no side effects related to the lytic agent or the Lysus Peripheral Catheter System. ${ }^{16}$ Although our success rate was not $100 \%$, the UAT group had a significantly higher success rate than did the CDT group. Refining our selection criteria for lytic therapy may help improve our success rates. Patients should also be educated regarding the signs and symptoms of bypass graft occlusion and encouraged to notify their vascular doctor as soon as possible in order to institute therapy earlier in the thrombotic process.

Short-term outcomes with the EKOS device in patients with lower extremity ischemia due to occlusions were evaluated by Schrijver et al., ${ }^{17}$ in a retrospective study. ${ }^{17}$ The EKOS group had a technical success rate of $97 \%$, radiologic success rate of $82 \%$, and clinical success rate of $77 \%$. Complete lysis was achieved in 24 hours or less. There were 9 re-interventions, and 2 of the 57 patients in the study required amputation within a 6-month period. Our amputation rate was significantly lower in the UAT group compared to our CDT group, but higher than the Schrijver study. To date, the Shrijver study is the largest cohort study of ultrasound-enhanced thrombolysis for occlusion of lower extremity arteries, and it demonstrated high initial success rate of ultrasound thrombolysis with a low complication rate. However, there was a considerable risk of recurrent ischemia in the 6 month follow-up period. Motarjeme evaluated lower extremity subacute arterial occlusions in 24 patients, resulting in a $100 \%$ technical success rate and $96 \%$ complete lysis rate ( 23 patients) with no hemorrhagic complications and only one re-occlusion during the 1 year follow-up period. ${ }^{12}$

Overall length of stay was shorter in the UAT group. Although we could not identify the precise determinant, an overall improved success rate, shorter treatment time, and less bleeding complication rate may have been contributing factors in the UAT group. This improved length of stay, however, did not translate to less direct costs for the admission. The overall higher costs for an operating room compared to an interventional radiology suite and higher reteplase costs for the UAT group may have contributed. Drug costs to the hospital for the lytic agent fluctuated during the study time period, potentially contributing to the differences in pharmacy costs. Additionally, the cost of an EKOS catheter are significantly higher compared to a standard multi-side hole catheter by $\$ 2,400$ in our institution. Also during the study time period, operating room costs were set much higher than radiology suite costs. Since then, our hospital has adjusted cost centers so that operating room and cardiac catheterization lab costs are similar when endovascular procedures are performed. Although, none of our patients experienced recurrent acute limb ischemia during the follow up period of 6months, data was not obtained beyond this time frame to evaluate long term outcomes. Another limitation of our retrospective review was the inability to clearly determine total amount of lytic drug utilized due to inconsistencies in record keeping at that time. Since the study, our institution has initiated system-wide electronic medical records, making recording and retrieval of drug amounts utilized more standard across the disciplines and easier to locate within the medical record. With the application of best practice guidelines, uniform order sets, and consistent post-procedural care, patients are receiving more standardized treatment across our institution, including the universal usage of ultrasound-directed over catheter-directed thrombolysis.

\section{Conclusion}

We have found that UAT has a higher success rate, shorter treatment time, shorter length of stay, higher limb salvage rate, and lower bleeding complication rate than CDT. However, costs, including total procedural and total admission direct costs were similar. Cost analyses of the various endovascular techniques, such as this endeavor, may help practitioners and institutions decide which minimally invasive technique to employ. Our institution has chosen to standardize techniques, including catheter choice (UAT), drug bolus and infusion amounts, and peri-procedural care in order to improve outcomes, decrease complications, and provide cost-effective care.

\section{Acknowledgments}

None.

\section{Conflicts of interest}

Author declares there is no conflicts opf interest.

\section{Funding}

None.

\section{References}

1. Blaisdell FW, Steele M, Allen RE. Management of lower extremity arterial ischaemia due to embolism and thrombosis. Surgery. 1978;84(6):822-834.

2. Earnshaw JJ, Whitman B, Foy C. National Audit of Thrombolysis for Acute Leg Ischemia (NATALI): clinical factors associated with early outcome. J Vasc Surg. 2004;39(5):1018-1025.

3. Cambria RP, Abbott WM. Acute arterial thrombosis of the lower extremity. Its natural history contrasted with arterial embolism. Arch Surg. 1984;119(7):784-787. 
4. Ouriel K, Veith FJ, Sasahara AA. Thrombolysis or peripheral arterial surgery: phase I results. TOPAS investigators. J Vasc Surg. 1996;23(1):64-73.

5. Francis CW, Blinc A, Lee S, et al. Ultrasound accelerates transport of recombinant tissue plasminogen activator into clots. Ultrasound Med Biol. 1995;21(3):419-424.

6. Soltani A, Singhal R, Garcia JL, et al. Absence of biological damage from prolonged exposure to intravascular ultrasound: A swine model. Ultrasonics. 2007;46(1):60-67.

7. Dumantepe M, Tarhan A, Yurdakul I, et al. US-accelerated catheterdirected thrombolysis for the treatment of deep venous thrombosis. Diag Interven Rad. 2013;19(3):251-258.

8. Owens CA. Ultrasound-enhanced thrombolysis: EKOS EndoWave infusion catheter system. Sem Interven Rad. 2008;25(1):37-41.

9. Dormandy J, Heeck L, Vig S. Acute limb ischemia. Semin Vasc Surg 1999;129(2):148-153.

10. Doormernik DE, Schrijver AM, Zeebregts CJ, et al. Advancements in catheter-directed ultrasound-accelereated thrombolysis. J Endovasc Ther. 2011;18(3):418-434.

11. Results of a prospective randomized trial evaluating surgery versus thrombolysis for ischemia of the lower extremity: the STILE trial. Ann Surg. 1994;220(3):251-268.
12. Motarjeme A. Ultrasound-enhanced Thrombolysis. J Endovasc Ther 2007; 14(2):251-256.

13. Schrijver AM, Reijnen MM, van Oostayen JA, et al. Dutch randomized controlled trial comparing standard catheter-directed thrombolysis versus ultrasound-accelerated thrombolysis for thromboembolic infrainguinal disease (DUET): design and rationale. Trials. 2011;12:20.

14. Medel R, Crowley RW, McKisic MS, et al. Sonothrombolysis: an emerging modality for the management of stroke. Neurosurgery. 2009;65(5):979-993.

15. Kuo WT, Gould MK, Louie JD, et al. Catheter-directed therapy for the treatment of massive pulmonary embolism: Systematic review and meta-analysis of modern techniques. $J$ Vasc Interven Rad. 2009;20(11):1431-1440.

16. Wissgott C, Richter A, Kamusella P, et al. Treatment of critical limb ischemia using ultrasound-enhanced thrombolysis (PARES Trial): final results. J Endovasc Ther. 2007;14(4):438-443.

17. Schrijver AM, Vos JA, Hoksbergen AW, et al. Ultrasound-accelerated thrombolysis for lower extremity ischemia: multicenter experience and literature review. J Cardiovasc Surg. 2001;53(4):467-476. 